\title{
Regular Parameter Elements and Regular Local Hyperrings
}

\author{
Hashem Bordbar (D) and Irina Cristea * (D) \\ Centre for Information Technologies and Applied Mathematics, University of Nova Gorica, \\ 5000 Nova Gorica, Slovenia; hashem.bordbar@ung.si \\ * Correspondence: irina.cristea@ung.si or irinacri@yahoo.co.uk; Tel.: +386-0533-15-395
}

Citation: Bordbar, H.; Cristea, I.

Regular Parameter Elements and

Regular Local Hyperrings.

Mathematics 2021, 9, 243.

https: / / doi.org/

$10.3390 /$ math 9030243

Academic Editor: Christos G. Massouros

Received: 31 December 2020

Accepted: 25 January 2021

Published: 26 January 2021

Publisher's Note: MDPI stays neutral with regard to jurisdictional clai$\mathrm{ms}$ in published maps and institutional affiliations.

Copyright: (C) 2021 by the authors. Licensee MDPI, Basel, Switzerland. This article is an open access article distributed under the terms and conditions of the Creative Commons Attribution (CC BY) license (https:// creativecommons.org/licenses/by/ $4.0 /)$.

\begin{abstract}
Inspired by the concept of regular local rings in classical algebra, in this article we initiate the study of the regular parameter elements in a commutative local Noetherian hyperring. These elements provide a deep connection between the dimension of the hyperring and its primary hyperideals. Then, our study focusses on the concept of regular local hyperring $R$, with maximal hyperideal $M$, having the property that the dimension of $R$ is equal to the dimension of the vectorial hyperspace $\frac{M}{M^{2}}$ over the hyperfield $\frac{R}{M}$. Finally, using the regular local hyperrings, we determine the dimension of the hyperrings of fractions.
\end{abstract}

Keywords: Krasner hyperring; prime/maximal hyperideal; length of hypermodules; regular local hyperring; parameter elements

\section{Introduction}

A regular local ring is known as a Noetherian ring with just one maximal ideal generated by $n$ elements, where $n$ is the Krull dimension of the ring. This is equivalent with the condition that the ring $R$ has a system of parameters that generate the maximal ideal, called regular system of parameters or regular parameter elements [1]. In commutative algebra, the theory of regular local rings plays a fundamental role and it has been developed since the late 30s and early 40s of the last century, thanks to the studies of two great mathematicians, Wolfgang Krull and Oscar Zariski. This happened just some years after that Frederic Marty introduced hypergroups as a generalization of groups in such a way that the single-valued group operation was extended to a hyperoperation, i.e., to a multi-valued operation. It is important to stress the fact that not all the properties of the group, such as the existence of the neutral element and the inverse, have been exactly transferred to the hypergroups, meaning that it is not obligatory that a hypergroup contains a neutral element or inverses. These requirements were requested later on, for so called canonical hypergroups, that were defined as the additive parts of the Krasner hyperrings and hyperfields $[2,3]$.

The hyperring is a hypercompositional structure endowed with one hyperoperation, namely the addition, and one binary operation, namely the multiplication, satisfying certain properties. Krasner [2] introduced the concept of hyperring for the first time in 1957 and investigated its applicability to the theory of valued fields. There are some other types of hyperrings: if the multiplication is a hyperoperation and the addition is a binary operation, we talk about the multiplicative hyperrings, defined by Rota [4]. If both, the addition and the multiplication, are hyperoperations with the additive part being a canonical hypergroup, then we have superrings [5], which were introduced by Mittas in 1973 [6]. Until now, the most well known and studied type of hyperrings is the Krasner hyperring, that has a plentitude of applications in algebraic geometry $[7,8]$, tropical geometry [9], theory of matroids [10], schemes theory [11], algebraic hypercurves [12,13], hypermomographies [14]. In addition, the theory of hypermodules was extensively investigated by Massouros [15]. In this article, the free and cyclic hypermodules are studied and several examples are provided such as the one obtained as a quotient of a P-module 
over a unitary ring P. Recently, Bordbar and his collaborators in [16-18] have introduced the length and the support of hypermodules and studied some properties of them, that are used also in this paper. Moreover, the connection between the hyperrings/hyperfields theory and geometry is very clearly explained by Massouros in [19]. This paper is a pylon in the current literature on algebraic hypercompositional algebra, because it describes the development of this theory (with a lot of examples and explanation of the terminology) from the first definition of hypergroup proposed by F. Marty to the hypergroups endowed with more axioms and used now a days.

In this paper, the theory of regular local rings is applied in the context of commutative Noetherian Krasner hyperrings, with only one maximal hyperideal, namely the local hyperrings. We first define the regular parameter elements in an arbitrary commutative local hyperring of finite dimension. These elements come up from the nice and deep relation existing between the dimension of a local hyperring with maximal hyperideal $M$ and the set of the generators of its $M$-primary hyperideals (see Theorem 4). More precisely, since in a local hyperring $R$ with maximal hyperideal $M$, the dimension of the ring $R$ is equal with the height of the hyperideal $M$, i.e., $\operatorname{dim} R=h t_{R} M$ as stated in Corolarry 1 , we can say that the regular parameter elements are a consequence of the investigation of the height of $M$ and the set of the generators for $M$-primary hyperideals in $R$. The other main objective of Section 3 is expressed by the result regarding the dimension of the quotient hyperrings (see Proposition 5). In Section 4 , using the local hyeprring $R$ with maximal hyperideal $M$ and the structure of the quotient hyperring, we introduce the hypermodule $\frac{M}{M^{2}}$ over the hyperrings $R$ and $\frac{R}{M}$, respectively. Since $\frac{R}{M}$ is a hyperfield, we conclude that $\frac{M}{M^{2}}$ is a vectorial hyperspace over the hyperfield $\frac{R}{M}$, as a direct consequence of Theorem 6 . Moreover, the investigation on the relation between the dimension of the hyperring $R$ (equivalently, the height $h t_{R} M$ of the maximal hyperideal $M$ ) and the dimension of the vectorial hyperspace $\frac{M}{M^{2}}$ conducts us to the definition of the regular local hyperrings. They are exactly local Noetherian hyperrings with the property that the maximal hyperideal $M$ can be generated by $d$ elements, where $d$ is the dimension of the hyperring. This follows from the main result of this section, i.e., Theorem 7 , saying that the dimension of a local Noetherian hyperring $R$ is the smallest number of elements that generate an $M$-primary hyperideal of $R$. Finally we apply these results in the class of the hyperrings of fractions, i.e., hyperrings of the form $R_{P}=S^{-1} R$, where $S=R \backslash P$, with $P$ a prime hyperideal of $S$, is a multiplicatively closed subset of $R$. We prove that the height of the prime hyperideal $P$ is equal to the height of the hyperideal $S^{-1} P$ in the hyperring of fractions $S_{P}$ (see Theorem 8 ). Final conclusions and some future works on this topic are gathered in the last section of the paper.

\section{Preliminaries}

In this section we collect some fundamental results regarding hyperrings, but for more details we refer the readers to [16-18]. Throughout the paper, $R$ denotes a Krasner hyperring, unless stated otherwise and we call it by short a hyperring. It was introduced by Krasner [2] as follows.

Definition 1. A (Krasner) hyperring is a hyperstructure $(R,+, \cdot)$ where

1. $(R,+)$ is a canonical hypergroup, i.e.,

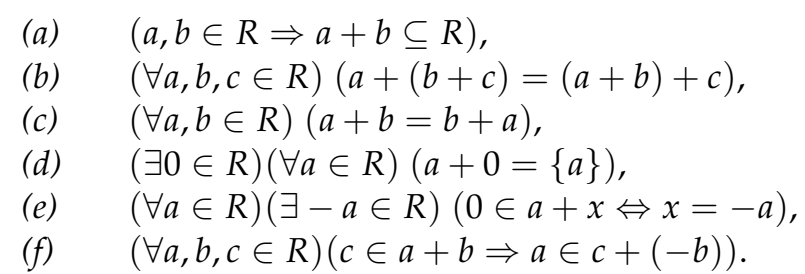

2. $(R, \cdot)$ is a semigroup with a bilaterally absorbing element 0 , i.e.,

(a) $(a, b \in R \Rightarrow a \cdot b \in R)$, 
(b) $(\forall a, b, c \in R)(a \cdot(b \cdot c)=(a \cdot b) \cdot c)$,

(c) $\quad(\forall a \in R)(0 \cdot a=a \cdot 0=0)$.

3. The product distributes from both sides over the sum

(a) $(\forall a, b, c \in R)(a \cdot(b+c)=a \cdot b+a \cdot c$ and $(b+c) \cdot a=b \cdot a+c \cdot a)$.

Moreover, if $(R, \cdot)$ is commutative, i.e.,

4. $(\forall a, b \in R)(a \cdot b=b \cdot a)$,

the hyperring is called commutative.

Definition 2. If every nonzero element in a hyperring $R$ with multiplicative identity 1 is invertible, i.e.,

(i) $\quad(\forall a \in R)\left(\exists a^{-1} \in R\right)\left(a \cdot a^{-1}=1_{R}\right)$,

then $R$ is called a hyper field.

Definition 3. A nonempty set I of a hyperring $R$ is called a hyperideal if, for all $a, b \in I$ and $r \in R$, we have $a-b \subseteq I$ and $a \cdot r \in I$. A proper hyperideal $M$ of a hyperring $R$ is called a maximal hyperideal of $R$ if the only hyperideals of $R$ that contain $M$ are $M$ itself and $R$. A hyperideal $P$ of a hyperring $R$ is called a prime hyperideal of $R$ if, for every pair of elements a and $b$ of $R$, the fact that $a b \in P$, implies either $a \in P$ or $b \in P$. In addition, a nonzero hyperring $R$ having exactly one maximal hyperideal is called a local hyperring.

Definition 4. A hyperring homomorphism is a mapping $f$ from a hyperring $R_{1}$ to a hyperring $R_{2}$ with units elements $1_{R_{1}}$ and $1_{R_{2}}$, respectively, such that

1. $(\forall a, b \in R)\left(f\left(a+R_{R_{1}} b\right)=f(a)+{ }_{R_{2}} f(b)\right)$.

2. $(\forall a, b \in R)\left(f\left(a \cdot R_{1} b\right)=f(a) \cdot R_{2} f(b)\right)$.

3. $f\left(1_{R_{1}}\right)=1_{R_{2}}$.

Definition 5. Let $f: R \rightarrow S$ be a hyperring homomorphism, $I$ be a hyperideal of $R$ and $J$ be a hyperideal of $S$.

(i) The hyperideal $\langle f(I)>$ of $S$ generated by the set $f(I)$ is called the extension of $I$ and it is denoted by $I^{e}$.

(ii) The hyperideal $f^{-1}(J)=\{a \in R \mid f(a) \in J\}$ is called the contraction of $J$ and it is denoted by $J^{c}$. It is known that, if $J$ is a prime hyperideal in $S$, then $J^{c}$ is a prime hyperideal in $R$.

Definition 6. A prime hyperideal $P$ of $R$ is called a minimal prime hyperideal over a hyperideal I of $R$ if it is minimal (with respect to inclusion) among all prime hyperideals of $R$ containing $I$. A prime hyperideal $P$ is called a minimal prime hyperideal if it is a minimal prime hyperideal over the zero hyperideal of $R$.

Definition 7. A hyperring $R$ is called Noetherian if it satisfies the ascending chain condition on hyperideals of $R$ : for every ascending chain of hyperideals $I_{1} \subseteq I_{2} \subseteq I_{3} \subseteq \ldots$ there exists $N \in \mathbb{N}$ such that $I_{n}=I_{N}$, for every natural number $n \geq N$ (this is equivalent to saying that every ascending chain of hyperideals has a maximal element). A hyperring $R$ is called Artinian if it satisfies the descending chain condition on hyperideals of $R$ : for every descending chain of hyperideals $I_{1} \supseteq I_{2} \supseteq I_{3} \supseteq$... there exists $N \in \mathbb{N}$ such that $I_{n}=I_{N}$, for every natural number $n \geq N$ (this is equivalent to saying that every descending chain of hyperideals has a minimal element).

Definition 8. Let $R$ be a hyperring with unit element 1. An R-hypermodule $M$ is a commutative hypergroup $(M,+)$ together with a map $R \times M \longrightarrow M$ defined by

$$
(a, m) \mapsto a \cdot m=a m \in M
$$

such that for all $a, b \in R$ and $m_{1}, m_{2} \in M$ we have: 
1. $(a+b) m_{1}=a m_{1}+b m_{1}$.

2. $\quad a\left(m_{1}+m_{2}\right)=a m_{1}+a m_{2}$.

3. $(a b) m_{1}=a\left(b m_{1}\right)$.

4. $a 0_{M}=0_{R} m_{1}=0_{M}$.

5. $1 m_{1}=m_{1}$, where 1 is the multiplicative identity in $R$.

Moreover, if $R$ is a hyperfield, then $M$ is called a vectorial hyperspace [20].

The next few results concern the concept of the radical of a hyperideal [16].

Definition 9. The radical of a hyperideal I of a hyperring $R$, denoted by $r(I)$, is defined as

$$
r(I)=\left\{x \mid x^{n} \in I, \text { for some } n \in \mathbb{N}\right\} .
$$

It can be proved that the radical of $I$ is the intersection of all prime hyperideals of $R$ containing $I$. In addition, a hyperideal $P$ in a hyperring $R$ is called primary if $P \neq R$ and the fact that $x y \in P$ implies either $x \in$ P or $y \in r(P)$.

Lemma 1. Let I be a hyperideal of the hyperring $R$, where $r(I)$ is a maximal hyperideal of $R$. Then $I$ is a primary hyperideal of $R$.

Proposition 1. Let $R$ be a Noetherian hyperring, $M$ a maximal hyperideal of $R$ and let $P$ be a hyperideal of $R$. Then the following statements are equivalent:

1. $\quad P$ is primary.

2. $r(P)=M$.

3. $M^{n} \subseteq P \subseteq M$, for some $n \in \mathbb{N}$.

Definition 10. Let $R$ be a commutative hyperring. An expression of the type

$$
P_{0} \subset P_{1} \subset \ldots \subset P_{n}
$$

(note the strict inclusions), where $P_{0}, \ldots, P_{n}$ are prime hyperideals of $R$, is called a chain of prime hyperideals of R; the length of such a chain is the number of the "links" between the terms of the chain, that is, 1 less than the number of prime hyperideals in the sequence.

Thus, the chain in (2) has length $n$. Note that, for a prime hyperideal $P$, we consider $P$ to be a chain, with just one prime hyperideal of $R$, of length 0 . Since $R$ is non-trivial, it contains at least one prime hyperideal, so there certainly exists at least one chain of prime hyperideals of $R$ of length 0 .

Definition 11. The supremum of the lengths of all chains of prime hyperideals of $R$ is called the dimension of $R$, denoted by $\operatorname{dim} R$.

Definition 12. Let $P$ be a prime hyperideal of a commutative hyperring $R$. The height of $P$, denoted by $h t_{R} P$, is defined to be the supremum of the lengths of all chains

$$
P_{0} \subset P_{1} \subset \ldots \subset P_{n}
$$

of prime hyperideals of $R$, for which $P_{n}=P$, if this supremum exists, and it is $\infty$, otherwise.

We conclude this preliminary section recalling the notion of vectorial hyperspace.

Definition 13 ([20]). Let $V$ be a vectorial hyperspace over a hyperfield $F$. A linear combination of vectors $v_{1}, v_{2}, \ldots, v_{n}$ is a set of the form $\sum_{i=1}^{n} r_{i} v_{i}$. Moreover, for a vectorial hyperspace $V$ over a hyperfield $F$ and $A \subseteq V, A$ is called linearly independent if for every finite set of vectors 
$\left\{v_{1}, v_{2}, \ldots, v_{n}\right\} \subseteq A, 0 \in \sum_{i=1}^{n} r_{i} v_{i}$ implies $r_{i}=0$ for $1 \leq i \leq n$. If $A$ is not linearly independent then $A$ is called linearly dependent.

Definition 14 ([20]). Let $V$ be a vectorial hyperspace over $F$. The set $A \subseteq V$ is called a spanning set if each vector of $V$ is contained in a linear combination of vectors from $A$. We say $V$ is the span of $A$ and write $V=\operatorname{Span}[A]$. In addition, a basis for a vectorial hyperspace $V$ is a subset $B$ of $V$ such that $B$ is both spanning and linearly independent set. A vectorial hyperspace is finite dimensional if it has a finite basis. Moreover, the number of the elements in an arbitrary basis of a vectorial hyperspace is called the dimension of the vectorial hyperspace, denoted here by vimV.

Note that we denote the dimension of the vectorial hyperspace by vdim $V$ (where "v" stays for "vectorial") in order to not confuse it with the dimension of a hyperring, denoted here as $\operatorname{dim} R$.

\section{Regular Parameter Elements in Local Hyperrings}

In this section, we introduce the notion of the regular parameter elements in a local hyperring and by using representative examples, we present some of their properties connected with the length and the dimension of the related vectorial hyperspace.

Definition 15. A unitary hyperring $R$ is called principal hyperideal hyperdomain when it has no zero divisors and all its hyperideals are generated by a single element.

M. Krasner had the great idea to construct hyperrings and hyperfields as quotients of rings and fields, respectively, called by him quotient hyperrings and quotient hyperfields, while this construction is known in literature as Krasner's construction [3]. The importance of these hyperrings and hyperfields in Krasner's studies is very clear explained by G. Massouros and Ch. Massouros in [19], as well as their different names given by some authors [7-11], with the risk of creating confusions. Therefore, in order to keep the original terminology, we recommend to read the papers of Nakasis [21] and Massouros [15,22,23].

Example $1([3,21])$. Let $(R,+, \cdot)$ be a ring and $G$ a normal subset of $R$ (which means that $r G=G r$ for every $r \in R$ ) such that $(G, \cdot)$ is a group and the unit element of $G$ is a unit element of $R$. Define an equivalence relation $\cong$ on $R$ as follows: $r \cong s$ if and only if $r G=s G$. Then, the equivalence class represented by $r$ is $P(r)=\{s \in R \mid s G=r G\}=r G$. Define now a hyperoperation $\oplus$ on the set of all equivalence classes $R / G$ as follows: $P(r) \oplus P(s)=\{P(t) \mid P(t) \cap(P(r)+P(s)) \neq \varnothing\}$ $=\left\{t G \mid \exists g_{1}, g_{2} \in G\right.$ such that $\left.t=r g_{1}+s g_{2}\right\}=\{t G \mid t G \subseteq r G+s G\}$, and define a binary operation on $R / G$ as $r G \cdot s G=r s G(P(r) \cdot P(s)=P(r s))$. Then, $(R / G, \oplus, \cdot)$ forms a hyperring. Moreover, if we choose $R$ to be a field, then we get that $(R / G, \oplus, \cdot)$ is a hyperfield.

Notice that the condition on the normality of $G$ can be substituted with a more general one, i.e., $r G s G=r s G$, for every $r, s \in R^{*}$, which is practically equivalent to the normality of $G$ only when the multiplicative semigroup is a group, so only when $R$ is a field, as Massouros proved in [22].

Proposition 2. Let $R$ be a principal hyperideal hyperdomain and let $p \in R-\{0\}$. Then the following statements are equivalent:

(i) $\quad p R$ is a maximal hyperideal of $R$.

(ii) $p R$ is a non-zero prime hyperideal of $R$.

Proof. $(i) \longrightarrow$ (ii) This implication is clear because $p \neq 0$ and every maximal hyperideal of a hyperring is also a prime hyperideal.

(ii) $\longrightarrow(i)$ Since $p R$ is a prime hyperideal of $R$, i.e., $p$ is not a unit element of $R$, we have $p R \subset R$. Let $I$ be a hyperideal of $R$ such that $p R \subseteq I \subset R$. Since $R$ is a principal hyperideal hyperdomain, there exists an element $a \in R$ such that $I=a R$. In addition, $a$ can not be a unit element because $I$ is a proper hyperideal. Since $R$ is a unitary hyperring, it follows that $p \in I$ and so $p=a b$ for some $b \in R$. Since $p R$ is a prime hyperideal, it follows 
that $p$ is irreducible and because $a$ is not a unit, we have that $b$ is a unit element of $R$. Thus, $p R=a R=I$ and therefore $p R$ is a maximal hyperideal.

Example 2 ([16]). Consider the set of integers $\mathbb{Z}$ and its multiplicative subgroup $G=\{-1,1\}$. Based on Example 1, the Krasner's construction $\frac{\mathbb{Z}}{G}$ is a principal hyperideal hyperdomain. In addition, the prime (also maximal) hyperideals of $\frac{\mathbb{Z}}{G}$ have the form $\langle p G\rangle$, where $p$ is a prime number. In $\frac{\mathbb{Z}}{G}$ we have $<0 \mathbb{Z}>\subset<2 \mathbb{Z}>$ a chain of prime hyperideals of length 1 . Since every nonzero prime hyperideal of $\frac{\mathbb{Z}}{G}$ is maximal, there does not exist a chain of prime hyperideals of $\frac{\mathbb{Z}}{G}$ of length 2 , therefore $\operatorname{dim} \frac{\mathbb{Z}}{G}=1$.

Remark 1. Let $R$ be a non-trivial commutative hyperring. By [16], every prime hyperideal of $R$ is contained in a maximal hyperideal of $R$ (and every maximal hyperideal is prime). Moreover, every prime hyperideal of $R$ contains a minimal prime hyperideal. It follows that dim $R$ is equal to the supremum of lengths of chains $P_{0} \subset P_{1} \subset \ldots \subset P_{n}$ of prime hyperideals of $R$, with $P_{n}$ a maximal and $P_{0}$ a minimal prime hyperideal. Indeed, if we have an arbitrary chain of prime hyperideals of $R$ with length $h$, like $P_{0}^{\prime} \subset P_{1}^{\prime} \subset \ldots \subset P_{h^{\prime}}^{\prime}$, then it is bounded above by the length of a special chain as it follows. If $P_{0}^{\prime}$ is not a minimal prime hyperideal, then another prime hyperideal can be inserted before it. On the other hand, if $P_{h}^{\prime}$ is not a maximal hyperideal of $R$, then another prime hyperideal can be inserted above it. Thus, if dimR is finite, then

$$
\begin{aligned}
\operatorname{dim} R & =\sup \left\{h t_{R} M \mid M \text { is a maximal hyperideal of } R\right\} \\
& =\sup \left\{h t_{R} P \mid P \text { is a prime hyperideal of } R\right\} .
\end{aligned}
$$

As a consequence, we have the following result.

Corollary 1. If $R$ is a local commutative hyperring with the maximal hyperideal $M$, then $\operatorname{dim} R=$ $h t_{R} M$.

The first property on the height of a hyperideal is highlighted in the next result.

Theorem 1 ([16]). Let $R$ be a commutative Noetherian hyperring and let $a \in R$ be a non-unit element. Let $P$ be a minimal prime hyperideal over the principal hyperideal $\langle a\rangle$ of $R$. Then, $h t_{R} P \leq 1$.

Now we can extend Theorem 1 to the case when $P$ is a minimal prime hyperideal over a hyperideal I generated not by one element, but by $n$ elements.

Theorem 2 ([16]). Let $R$ be a commutative Noetherian hyperring. Suppose that I is a proper hyperideal of $R$ generated by $n$ elements and $P$ is a minimal prime hyperideal over $I$. Then, $h t_{R} P \leq n$.

Lemma 2. In a commutative Noetherian hyperring $R$, let I be a hyperideal and $P$ a prime hyperideal of $R$, such that $I \subseteq P$ and $h t_{R} I=h t_{R} P$. Then, $P$ is a minimal prime hyperideal over $I$.

Proof. Suppose that $P$ is not a minimal prime hyperideal over $I$. Then, by Remark 1 , there exists a prime hyperideal $Q$ of $R$ which is minimal and $I \subseteq Q \subset P$. Thus, $h t_{R} I \leq h t_{R} Q<$ $h t_{R} P$, obtaining a contradiction. So $P$ is a minimal prime hyperideal over $I$.

The next theorem states the conditions under which there exists a proper hyperideal of height $n$ and generated by $n$ elements.

Theorem 3 ([17]). Let $R$ be a commutative Noetherian hyperring and $P$ a prime hyperideal of $R$, with $h t_{R} P=n$. Then there exists a proper hyperideal I of $R$ having the following properties:

(i) $I \subseteq P$. 
(ii) I is generated by $n$ elements.

(iii) $h t_{R} I=n$.

We have now all the elements to determine the dimension of a commutative local Noetherian hyperring.

Theorem 4. Suppose that $R$ is a commutative local Noetherian hyperring, having $M$ as its unique maximal hyperideal. Then, $\operatorname{dim} R$ is equal to the smallest number of elements of $R$ that generate an M-primary hyperideal. In other words,

$$
\operatorname{dim} R=\min \left\{i \in \mathbb{N}, \exists a_{1}, a_{2}, \ldots, a_{i} \in R, \sum_{j=1}^{i} R a_{j} \text { is an } M \text { - primary hyperideal }\right\} .
$$

Proof. Let

$$
d=\min \left\{i \in \mathbb{N}, \exists a_{1}, a_{2}, \ldots, a_{i} \in R, \sum_{j=1}^{i} R a_{j} \text { is an } M \text { - primary hyperideal }\right\} .
$$

By using Corollary 1 and Theorem 2, we have $\operatorname{dim} R=h t_{R} M \leq d$, because an $M$ primary hyperideal must have $M$ as its minimal prime hyperideal. On the other hand, by using Lemma 2 and Theorem 3, there exists a hyperideal $P$ of $R$ which has $M$ as a minimal prime hyperideal and which can be generated by $\operatorname{dim} R=h t_{R} M$ elements. In addition, every prime hyperideal of $R$ is contained in $M$. Hence, $M$ must be the only one associated prime hyperideal of $P$. Thus, $P$ is an $M$-primary hyperideal.

As a result that there exists an $M$-primary hyperideal of $R$ which can be generated by $\operatorname{dim} R$ elements, we have $d \leq \operatorname{dim} R$. Therefore, $d=\operatorname{dim} R$ and this completes the proof.

Definition 16. Let $R$ be a local hyperring of dimension d, with $M$ as its unique maximal hyperideal. By regular parameter elements of $R$ we mean a set of $d$ elements of $R$ that generate an $M$-primary hyperideal of $R$.

Remark 2. Based on Theorem 4, we conclude that each local hyperring of dimension at least 1 possesses a set of regular parameter elements.

Example 3. On the set $R=\{0,1,2\}$ define the hyperaddition + and the multiplication $\cdot$ by the

\begin{tabular}{|c|c|c|c|c|c|c|}
\hline+ & 0 & 1 & 2 & & 0 & 1 \\
\hline 0 & 0 & 1 & 2 & 0 & 0 & 0 \\
\hline 1 & 1 & $R$ & 1 & 1 & 0 & 1 \\
\hline 2 & 2 & 1 & $M$ & 2 & 0 & 2 \\
\hline
\end{tabular}
following tables

Then, $R$ is a commutative local Noetherian hypering and $M=\{0,2\}$ is the only maximal hyperideal of $R$. Thus, $\operatorname{dim} R=h t_{R} M$ and $M$ is an M-primary hyperideal. One can check that $h t_{R} M=0$, so $\operatorname{dim} R=0$. Therefore this hyperring has no regular parameter elements.

Proposition 3. Let $R$ be a local hyperring of dimension $d$, with the unique maximal hyperideal $M$, and let $a_{1}, a_{2}, \ldots, a_{d}$ be a set of regular parameter elements for $R$. For any $n_{1}, n_{2}, \ldots, n_{d} \in \mathbb{N}$, we have that $a_{1}^{n_{1}}, a_{2}^{n_{2}}, \ldots, a_{d}^{n_{d}}$ form a set of regular parameter elements for $R$, too.

Proof. The proof is straightforward. 
Proposition 4. Let $f: R \rightarrow S$ be a surjective homomorphism of commutative hyperrings. Suppose that $Q_{1}, Q_{2}, \ldots, Q_{n}, P_{1}, P_{2}, \ldots, P_{n}$ are hyperideals of $R$, all of which containing Kerf $f$, with $r\left(Q_{i}\right)=P_{i}$, for $i=1,2, \ldots, n$. Then

$$
I=Q_{1} \cap Q_{2} \cap \ldots \cap Q_{n}
$$

is a (minimal) primary decomposition of I if and only if

$$
I^{e}=Q_{1}^{e} \cap Q_{2}^{e} \cap \ldots \cap Q_{n}^{e}
$$

with $r\left(Q_{i}^{e}\right)=P_{i}^{e}$ for $i=1,2, \ldots, n$ is a (minimal) primary decomposition of $I^{e}$.

Proof. The proof is straightforward.

We can conclude that $I$ is a decomposable hyperideal of $R$ (meaning that it has a primary decomposition) if and only if $I^{e}$ is a decomposable hyperideal of $S$. Moreover, if

$$
I=Q_{1} \cap Q_{2} \cap \ldots \cap Q_{n}
$$

where $r\left(Q_{i}\right)=P_{i}$, for $i=1,2, \ldots, n$, then we call the set $\left\{P_{1}, P_{2}, \ldots, P_{n}\right\}$, the set of the associated prime hyperideals related to I and denoted by $\operatorname{ass}_{R} I$. Using Proposition 4 , for the hyperideal $I^{e}$ of $S$ the related associated prime hyperideals form the set

$$
\operatorname{ass}_{S} I^{e}=\left\{P^{e} \mid P \in \operatorname{ass}_{R} I\right\}=\left\{P_{1}^{e}, P_{2}^{e}, \ldots, P_{n}^{e}\right\} .
$$

Corollary 2. Let I be a proper hyperideal of the commutative hyperring $R$. Using the canonical hyperring homomorphism from $R$ to the quotient $\frac{R}{I}$, we conclude that if $J$ is a hyperideal of $R$ such that $I \subseteq J$, then $J$ is a decomposable hyperideal of $R$ if and only if $\frac{I}{I}$ is a decomposable hyperideal of $\frac{R}{T}$, and we have

$$
\operatorname{ass}_{\frac{R}{I}}\left(\frac{J}{I}\right)=\left\{\frac{P}{I}: P \in \operatorname{ass}_{R} J\right\}
$$

Theorem 5 ([17]). Let $R$ be a commutative Noetherian hyperring, $P$ a prime hyperideal of $R$ and $I$ a proper hyperideal of $R$ generated by $n$ elements, such that $I \subseteq P$. Then:

$$
h t_{\frac{R}{T}} \frac{P}{I} \leq h t_{R} P \leq h t_{\frac{R}{I}} \frac{P}{I}+n .
$$

Proposition 5. Let $R$ be a local commutative hyperring of dimention $d$ with its maximal hyperideal $M$, and let $a_{1}, a_{2}, \ldots, a_{t} \in M$. Then,

$$
\operatorname{dim} R-t \leq \operatorname{dim} R /\left(a_{1}, a_{2}, \ldots, a_{t}\right) \leq \operatorname{dim} R .
$$

Moreover, $\operatorname{dim} R /\left(a_{1}, a_{2}, \ldots, a_{t}\right)=\operatorname{dim} R-t$ if and only if $a_{1}, a_{2}, \ldots, a_{t}$ are all distinct elements and form a set of regular parameter elements of $R$.

Proof. Using Corollary 1 , we have $\operatorname{dim} R=h t_{R} M$ and therefore

$$
\operatorname{dimR} /\left(a_{1}, a_{2}, \ldots, a_{t}\right)=h t_{R /\left(a_{1}, a_{2}, \ldots, a_{t}\right)} M /\left(a_{1}, a_{2}, \ldots, a_{t}\right) .
$$

By using Theorem 5, we conclude that

$$
\operatorname{dim} R-t \leq \operatorname{dim} R /\left(a_{1}, a_{2}, \ldots, a_{t}\right) \leq \operatorname{dim} R .
$$

Now let can $: R \rightarrow R /\left(a_{1}, a_{2}, \ldots, a_{t}\right)$ be the canonical hyperring homomorphism such that $\operatorname{can}(r)=r+\left(a_{1}, a_{2}, \ldots, a_{t}\right)$. Set $\bar{M}=\frac{M}{\left(a_{1}, a_{2}, \ldots, a_{t}\right)}$.

Suppose that $\operatorname{dim} R /\left(a_{1}, a_{2}, \ldots, a_{t}\right)=d-t$. Then, $t \leq d$, and by using Theorem 4 , there exist $\overline{a_{t+1}}, \overline{a_{t+2}}, \ldots, \overline{a_{d}} \in R /\left(a_{1}, a_{2}, \ldots, a_{t}\right)$, where $\overline{a_{j}}=a_{j}+\left(a_{1}, a_{2}, \ldots, a_{t}\right)$ for $j=$ 
$t+1, \ldots, d$, such that $\left(\overline{a_{t+1}}, \overline{a_{t+2}}, \ldots, \overline{a_{d}}\right)$ is an $\bar{M}$-primary hyperideal of $R /\left(a_{1}, a_{2}, \ldots, a_{t}\right)$. Therefore, $a_{t+1}, a_{t+2}, \ldots, a_{d} \in M$. Thus,

$$
\frac{\left(a_{1}, a_{2}, \ldots, a_{t}, a_{t+1}, \ldots, a_{d}\right)}{\left(a_{1}, a_{2}, \ldots, a_{t}\right)}
$$

is an $\bar{M}$-primary hyperideal of $R /\left(a_{1}, a_{2}, \ldots, a_{t}\right)$. By using Proposition 4 and Corollary 2, we conclude that $\left(a_{1}, a_{2}, \ldots, a_{d}\right)$ is an $M$-primary hyperideal of $R$. It now follows from Theorem 4 , that $a_{1}, a_{2}, \ldots, a_{d}$ are all distinct and $\left\{a_{1}, a_{2}, \ldots, a_{d}\right\}$ is a set of regular parameter elements of $R$. Therefore, $a_{1}, a_{2}, \ldots, a_{t}$ are all distinct and form a set of regular parameter elements of $R$.

Now suppose that $t \leq d$ and there exist $a_{t+1}, \ldots, a_{d} \in M$ such that

$$
a_{1}, a_{2}, \ldots, a_{t}, a_{t+1}, \ldots, a_{d}
$$

form a set of regular parameter elements of $R$. Thus, $\left(a_{1}, a_{2}, \ldots, a_{d}\right)$ is an M-primary hyperideal of $R$. Therefore, by using Proposition 4 and Corollary $2,\left(\overline{a_{t+1}}, \overline{a_{t+2}}, \ldots, \overline{a_{d}}\right)$ is an $\bar{M}$-primary hyperideal of $R /\left(a_{1}, a_{2}, \ldots, a_{t}\right)$. Thus, by using Theorem 4 , we have $d-t \geq \operatorname{dim} \bar{R}$. But, it follows from the first part that $d-t \leq \operatorname{dim} \bar{R}$, and so the proof is complete.

\section{Regular Local Hyperrings}

The aim of this section is to define the regular local hyperrings. For doing this, we will first prove that, in a local Noetherian hyperring $R$ with the unique maximal hyperideal $M$, the $R$-hypermodule $\frac{M}{M^{2}}$ is a vectorial hyperspace over the hyperfield $\frac{R}{M}$. Then we will establish a relation between the dimension of the hyperring $R$ and the dimension of the vectorial hyperspace $\frac{M}{M^{2}}$. Finally, we will present a new characterization of the dimension of the hyperring of fractions.

Definition 17 ([18]). Let $M$ be a hypermodule over the commutative hyperring $R, N$ be a subhypermodule of $M$ and $I \subseteq M$ with $I \neq 0$. We define the hyperideal

$$
(N: R I)=\{r \in R \mid r \cdot x \in N \text { for all } x \in I\} .
$$

For any element $m \in M$, we denote $\left(N:_{R} m\right)$ instead of $\left(N:_{R}\{m\}\right)$. In addition, in the special case when $N=0$, the hyperideal

$$
\left(0:_{R} I\right)=\{r \in R \mid r \cdot x=0 \text { for all } x \in I\}
$$

is called the annihilator of $I$ and is denoted by $A n n_{R}(I)$. Moreover, for any element $m \in M$, we call the hyperideal $\left(0:_{R} m\right)$ the annihilator of the element $m$.

Example 4. Let us continue with Example 3, where $R$ is an R-hypermodule and its hyperideal $M$ is a subhypermodule. We have

$$
\operatorname{Ann}_{R}(M)=\left(0:_{R} M\right)=\{r \in R \mid r \cdot m=0 \text { for all } m \in M\}=M .
$$

Moreover, for $m=2$ we have

$$
\left(0:_{R} 2\right)=\{r \in R \mid r \cdot 2=0\}=\{0,2\} .
$$

Proposition 6. Let $M$ be a hypermodule over the commutative hyperring $R$ and $I$ be a hyperideal of $R$ such that $I \subseteq A n n_{R}(M)$. Then, $M$ is a hypermodule over $\frac{R}{I}$.

Proof. Suppose that $r, r^{\prime} \in R$ such that $r+I=r^{\prime}+I$. We have $r-r^{\prime} \subseteq I \subseteq A n n_{R}(M)$, and so $\left(r-r^{\prime}\right) m=0$ for any arbitrary element $m \in M$. Thus, $r m=r^{\prime} m$. Hence, we can 
define a mapping $\frac{R}{I} \times M \longrightarrow M$ such that $(r+I, m) \longrightarrow r m$. It is a routine to check that $M$ has an $\frac{R}{I}$-hypermodule structure.

Proposition 7. Let $R$ be a local hyperring with its maximal hyperideal $M$ and consider the hyperfield $F=\frac{R}{M}$. Let $N$ be a finitely generated $R$-hypermodule. Then the $R$-hypermodule $\frac{N}{M N}$ has a natural structure as a hypermodule over $\frac{R}{M}$ as an F-vectorial hyperspace.

Moreover, let $n_{1}, n_{2}, \ldots, n_{t} \in N$. Then the following statements are equivalent.

(i) $N$ is generated by $n_{1}, n_{2}, \ldots, n_{t}$.

(ii) The R-hypermodule $\frac{N}{M N}$ is generated by $n_{1}+M N, n_{2}+M N, \ldots, n_{t}+M N$.

(iii) The F-vectorial hyperspace $\frac{N}{M N}$ is generated by $n_{1}+M N, n_{2}+M N, \ldots, n_{t}+M N$.

Proof. Since the $R$-hypermodule $\frac{N}{M N}$ is annihilated by $M$, i.e., $M \subseteq A n n_{R}\left(\frac{N}{M N}\right)$, by using Proposition 6, it has a natural structure as hypermodule over $\frac{R}{M}$. In addition, since $F=\frac{R}{M}$ is a hyperfield, $\frac{N}{M N}$ is also an $F$-vectorial hyperspace.

It is clear that $(i)$ implies (ii).

The $R$-hypermodule and $F$-vectorial hyperspace structures of $\frac{N}{M N}$ are related by the formula

$$
r(n+M N)=(r+M)(n+M N)
$$

for all $r \in R$ and $n \in N$. Thus, the equivalence of statements (ii) and (iii) is clear.

It remains to prove that $(i i)$ implies $(i)$. Assume that $(i i)$ holds, so the $R$-hypermodule $\frac{N}{M N}$ is generated by the elements

$$
n_{1}+M N, n_{2}+M N, \ldots, n_{t}+M N
$$

Let $G=R n_{1}+R n_{2}+\ldots+R n_{t}$. First, we will show that $N=G+M N$. Let $n \in N$. Then there exist $r_{1}, r_{2}, \ldots, r_{t} \in R$ such that

$$
n+M N=r_{1}\left(n_{1}+M N\right)+r_{2}\left(n_{2}+M N\right)+\ldots+r_{t}\left(n_{t}+M N\right) .
$$

Hence, $n-\sum_{i=1}^{n} r_{i} n_{i} \in M N$. It follows that $N \subseteq G+M N$. On the other side, it is clear that $G+M N \subseteq N$. Thus, we have $N=G+M N$. Now by using Corollary 2.9 in [16], we conclude that $N=G$, so $N$ is generated by the elements $n_{1}, n_{2}, \ldots, n_{t}$.

Theorem 6. The F-vectorial hyperspace $\frac{N}{M N}$ in Proposition 7 has finite dimension and the number of the elements in each minimal generating set for the R-hypermodule $N$ is equal to vdim $\frac{N}{M N}$.

Proof. Since $N$ is a finitely generated $R$-hypermodule, it follows from Proposition 7 that $\frac{N}{M N}$ is a finitely generated $F$-vectorial hyperspace. Therefore, its dimension is finite. the set

Let $\left\{n_{1}^{\prime}, n_{2}^{\prime}, \ldots, n_{p}^{\prime}\right\}$ be a minimal generating set for $N$. By Proposition 7 , we know that

$$
\left\{n_{1}^{\prime}+M N, n_{2}^{\prime}+M N, \ldots, n_{p}^{\prime}+M N\right\}
$$

is a generating set for the $F$-vectorial hyperspace $\frac{N}{M N}$ and since $\left\{n_{1}^{\prime}, n_{2}^{\prime}, \ldots, n_{p}^{\prime}\right\}$ is a minimal generating set for $N$, it follows that no proper subset of $\left\{n_{1}^{\prime}+M N, n_{2}^{\prime}+M N, \ldots, n_{p}^{\prime}+M N\right\}$ generates $\frac{N}{M N}$. Thus, the set $\left\{n_{1}^{\prime}+M N, n_{2}^{\prime}+M N, \ldots, n_{p}^{\prime}+M N\right\}$ is a basis for the $F$ vectorial hyperspace $\frac{N}{M N}$ and so vdim $\frac{N}{M N}=p$.

Note that the $R$-hypermodule $\frac{M}{M^{2}}$ is annihilated by $M$. If $R$ is a Noetherian hyperring, then by Proposition $6, \frac{M}{M^{2}}$ has a natural structure as a vectorial hyperspace over the hyperfield $\frac{R}{M}$. In addition, using Proposition 7, the dimension of the vectorial hyperspace $\frac{M}{M^{2}}$ is equal to the number of the elements in an arbitrary minimal generating set for $M$. Therefore, we have the following result. 
Theorem 7. Let $R$ be a local Noetherian hyperring with maximal hyperideal $M$. Then,

$$
\operatorname{dim} R \leq \operatorname{vim}_{\frac{R}{M}} \frac{M}{M^{2}}
$$

Proof. The inequality is clear because $M$ is an $M$-primary hyperideal of the hyperring $R$ and as mentioned before $v \operatorname{dim}_{\frac{R}{M}} \frac{M}{M^{2}}$ is the number of the elements of an arbitrary minimal generating set for $M$. Based on Theorem $4, \operatorname{dim} R$ is the smallest number of elements that generate an $M$-primary hyperideal of $R$.

Definition 18. Let $R$ be a local Noetherian hyperring with maximal hyperideal M. Then, $R$ is called a regular hyperring when $\operatorname{dim} R=\operatorname{vim}_{\frac{R}{M}} \frac{M}{M^{2}}$.

Remark 3. For a local Noetherian hyperring $R$ with one maximal hyperideal $M$ and $\operatorname{dim} R=d$, we have the following statements:

(i) The dimension of the $\frac{R}{M}$-vectorial hyperspace $\frac{M}{M^{2}}$ is the number of the elements in each minimal generating set for the hyperideal M. By using Theorem 4, at least d elements are needed to generate $M$, and $R$ is a regular hyperring when the hyperideal $M$ can be generated by exactly $d$ elements.

(ii) Suppose that $R$ is a regular hyperring and $a_{1}, a_{2}, \ldots, a_{d} \in M$. By using Proposition 7 , the elements $a_{1}, a_{2}, \ldots, a_{d}$ generate $M$ if and only if $a_{1}+M^{2}, a_{2}+M^{2}, \ldots, a_{d}+M^{2}$ in $\frac{M}{M^{2}}$ form a basis for this $\frac{R}{M}$-vectorial hyperspace, equivalently if and only if $a_{1}+M^{2}, a_{2}+M^{2}, \ldots, a_{d}+$ $M^{2}$ form a linearly independent set.

We conclude this section with a new characterization of the dimension of the hyperring of fractions $R_{P}=S^{-1} R$, where $S=R \backslash P$, with $P$ a prime hyperideal of $S$, is a multiplicatively closed subset of $R$. As proved in [16,17], $R_{P}$ is a local Noetherian hyperring. First, we recall the main properties of the hyperring of fractions.

Proposition 8 ([16]). Let $S$ be a multiplicatively closed subset of a hyperring $R$.

(i) Every hyperideal in $S^{-1} R$ is an extended hyperideal.

(ii) If $I$ is a hyperideal in $R$, then $I^{e}=S^{-1} R$ if and only if $I \cap S=\varnothing$.

(iii) A hyperideal $I$ is a contracted hyperideal of $R$ if and only if no element of $S$ is a zero divisor in $R / I$.

(iv) The prime hyperideals of $S^{-1} R$ are in one-to-one correspondence with the prime hyperideals of $R$ that don't meet $S$, with the correspondence given by $P \leftrightarrow S^{-1} P$.

Theorem 8. Let $S$ be a multiplicatively closed subset of $R$ and $P$ be a prime hyperideal of $R$ such that $P \cap S=\varnothing$. Then, $h t_{R} P=h t_{S^{-1} R} S^{-1} P$.

Proof. By Proposition 8 , it follows that $S^{-1} P$ is a prime hyperideal of $S^{-1} R$. Let

$$
P_{0} \subset P_{1} \subset \ldots \subset P_{n}=P
$$

be a chain of prime hyperideals of $R$. Again by Proposition 8 , it follows that

$$
P_{0}^{e} \subset P_{1}^{e} \subset \ldots \subset P_{n}^{e}
$$

is a chain of prime hyperideals of $S^{-1} R$ with $P_{n}^{e}=P^{e}=S^{-1} P$, and therefore $h t_{R} P \leq$ $h t_{S^{-1} R} S^{-1} P$. On the other side, if

$$
Q_{0} \subset Q_{1} \subset \ldots \subset Q_{n}
$$


is a chain of prime hyperideals of $S^{-1} R$ with $Q_{n}=P^{e}$, then using Propositions 8, we get that

$$
Q_{0}^{c} \subset Q_{1}^{c} \subset \ldots \subset Q_{n}^{c}
$$

is a chain of prime hyperideals of $R$ with $Q_{n}^{c}=P^{e c}=P$. So we have $h t_{S^{-1}} S^{-1} P \leq h t_{R} P$. Therefore, it follows that $h t_{R} P=h t_{S^{-1} R} S^{-1} P$.

Combining the previous results, we get now the following important consequence.

Corollary 3. For a prime hyperideal $P$ of a commutative hyperring $R$, it follows that

$$
h t_{R} P=h t_{R_{P}} S^{-1} P=\operatorname{dim}\left(R_{P}\right) .
$$

In the following we will illustrate the previous results by several examples.

Example 5. Let $R$ be a commutative Noetherian hyperring. Suppose that there exists a prime hyperideal $P$ of $R$ with $h t P=n$ and that can be generated by $n$ elements $a_{1}, a_{2}, \ldots, a_{n}$. Consider the localisation hyperring $R_{P}$, that is a local hyperring. According with Theorem 8 and Corollary 3 , it has dimension $n$. Since the dimension of localization hyperring $R_{P}$ is $n$, the maximal hyperideal of this hyperring, i.e.,

$$
P R_{P}=\left(\sum_{i=1}^{n} R a_{i}\right) R_{P}=\sum_{i=1}^{n} R_{P} \frac{a_{i}}{1}
$$

can be generated by $n$ elements, that are also regular parameter elements, it follows that $R_{P}$ is a regular hyperring.

Example 6. Let $p$ be a prime number. Based on Example 2, we have $h t_{\mathbb{Z}} p \mathbb{Z}=1$. Since $p \mathbb{Z}$ is a prime hyperideal of $\mathbb{Z}$ which can be generated by one element, it follows from Example 5 that $\mathbb{Z}_{p \mathbb{Z}}$ is a regular hyperring of dimension 1 and $p$ is a regular parameter element.

Example 7. Let $R$ be a principal hyperideal hyperdomain which is not a hyperfield, and let $M$ be a maximal hyperideal of $R$. By using Proposition 2, $M$ is a prime hyperideal of $R$ and also principal hyperideal with height 1. Using Example 5, it follows that $R_{M}$ is a regular hyperring of dimension 1.

\section{Conclusions}

In this paper, we have to define the regular parameter elements in a commutative local Noetherian hyperring $R$ with maximal hyperideal $M$ and present some properties related to them. After investigating some results concerning the quotient hypermodule $\frac{M}{M^{2}}$ over the hyperfield $\frac{R}{M}$, our study has focused on regular local hyperrings. We have studied the relation between the dimension of a commutative local Noetherian hyperring and the dimension of the vectorial hyperspace $\frac{M}{M^{2}}$ over the hyperfield $\frac{R}{M}$.

Our future work will include new results regarding the regular local hyperrings. In particular we will investigate whether they are hyperdomains or not. In addition, we will study the properties of the hyperideals generated by a subset of the regular parameter elements and the relation between the length of these hyperideals and the dimension of the hyperring.

Author Contributions: Conceptualization, H.B. and I.C.; methodology, H.B. and I.C.; investigation, H.B. and I.C.; writing—original draft preparation, H.B.; writing—review and editing, I.C.; funding acquisition, I.C. Both authors have read and agreed to the published version of the manuscript.

Funding: The second author acknowledges the financial support from the Slovenian Research Agency (research core funding No. P1-0285).

Conflicts of Interest: The authors declare no conflict of interest. 


\section{References}

1. Kemper, G. Regular local rings. In A Course in Commutative Algebra; Graduate Texts in Mathematics; Springer: Berlin/ Heildeberg, Germany, 2011; Volume 256.

2. Krasner, M. Approximation des Corps Values Complets de Caracteristique $p, p>0$, Par ceux de Caracteristique Zero; Colloque d' Algebre Superieure (Bruxelles, Decembre 1956); CBRM: Bruxelles, Belgium, 1957.

3. Krasner, M. A class of hyperrings and hyperfields. Int. J. Math. Math. Sci. 1983, 6, 307-312. [CrossRef]

4. Rota, R. Strongly distributive multiplicative hyperrings. J. Geom. 1990, 1-2, 130-138. [CrossRef]

5. Ameri, R.; Eyvazi, M.; Hoskova-Mayerova, S. Superring of Polynomials over a Hyperring. Mathematics 2019, 7, 902. [CrossRef]

6. Mittas, J.D. Sur certaines classes de structures. hypercompositionnelles. Proc. Acad. Athens 1973, 48, $298-318$.

7. Connes, A.; Consani, C. On the notion of geometry over $\mathbf{F}_{1}$. J. Algebraic Geom. 2011, 20, 525-557. [CrossRef]

8. Jun, J. Algebraic geometry over hyperrings. Adv. Math. 2018, 323, 142-192. [CrossRef]

9. Viro, O. Hyperfields for tropical geometry I. Hyperfields and dequantization. arXiv 2010, arXiv:1006.3034.

10. Baker, M.; Bowler, N. Matroids over partial hyperstructures. Adv. Math. 2019, 343, 821-863. [CrossRef]

11. Jun, J. Hyperstructures of affine algebraic group schemes. J. Number Theory 2016, 167, 336-352. [CrossRef]

12. Vahedi, V.; Jafarpour, M.; Aghabozorgi, H.; Cristea, I. Extension of elliptic curves on Krasner hyperfields. Comm. Algebra 2019, 47, 4806-4823. [CrossRef]

13. Vahedi, V.; Jafarpour, M.; Hoskova-Mayerova, S.; Aghabozorgi, H.; Leoreanu-Fotea, V.; Bekesiene, S. Derived Hyperstructures from Hyperconics. Mathematics 2020, 8, 429. [CrossRef]

14. Vahedi, V.; Jafarpour, M.; Cristea, I. Hyperhomographies on Krasner hyperfields. Symmetry 2019, 11, 1442. [CrossRef]

15. Massouros, C.G. Free and cyclic hypermodules. Ann. Mat. Pura Appl. 1988, 150, 153-166. [CrossRef]

16. Bordbar, H.; Cristea, I. Height of prime hyperideals in Krasner hyperrings. Filomat 2017, 31, 6153-6163. [CrossRef]

17. Bordbar, H.; Cristea, I.; Novak, M. Height of hyperideals in Noetherian Krasner hyperrings. Politehn. Univ. Bucharest Sci. Bull. Ser. A Appl. Math. Phys. 2017, 79, 31-42.

18. Bordbar, H.; Novak, M.; Cristea, I. A note on the support of a hypermodule. J. Algebra Appl. 2020, 19, 2050019. [CrossRef]

19. Massouros, G.; Massouros, C. Hypercompositional Algebra, Computer Science and Geometry. Mathematics 2020, 8, 1338. [CrossRef]

20. Mittas, J. Espaces vectoriels sur un hypercorps, Introduction des hyperspaces affines et Euclidiens. Math. Balk. 1975, 5, 199-211.

21. Nakassis, A. Recent results in hyperring and hyperfield theory. Int. J. Math. Math. Sci. 1988, 11, 209-220. [CrossRef]

22. Massouros, C.G. On the theory of hyperrings and hyperfields. Algebra Logika 1985, 24, 728-742. [CrossRef]

23. Massouros, C.G. Constructions of hyperfields. Math. Balk. 1991, 5, 250-257. 